\title{
The Transmission Problem to Thermoelastic Plate of Hyperbolic Type*
}

\author{
Juan C. Vila Bravo Jaime E. Muñoz Rivera
}

\begin{abstract}
In this paper we consider the thermoelastic plate equation with localized thermal dissipation of memory type, proposed by Gurtin and Pipkin [3]. We will show that the solution of the corresponding model decays exponentially as time goes to infinity, provided the relaxation function decays exponentially. The mean difference with others thermoelastic system is that the whole system is of hyperbolic type, and the dissipation is weaker (indefinite) than such given by the Fourier Law for the heat transmission.
\end{abstract}

Keywords - Exponential stability, transmission problem, thermoelasticity.

\section{Introduction}

In classical linear theory of thermoelasticity the Fourier's law is used to describe the heat conduction of the body. Therefore, the corresponding thermoelastic system consist on an hyperbolic equation for the displacemente field acoupled with a parabolic equation for the heat equation. This theory has two shortcomings: First, it is unable to take into account the memory effect which may prevail in some materials, particularly at low temperatures. Second, the corresponding parabolic part of the system predicts an unrealistic result, the thermal disturbance at one point of the body is instantly felt everywhere in the body. To attend this problems Gurtin and Pipkin [3] introduce a new constitutive law for the heat flux. This constitutive law depends on the heat memory and as a first consequence the parabolicity of the system is removed. Therefore the thermoelastic system is fully hyperbolic. So we have finite speed of propagation (see [7]).

In this paper we study the transmission problem for a partial thermoelastic plate. That is, we consider a plate composed by two components: One of them is a thermoelastic part and the other an elastic part insensible to changes of temperature. This in particular means that the thermal constant are discontinuous on the plate. Positive over the thermoelastic region and zero over the elastic part. So, the corresponding plate system has discontinuous coefficients.

More precissally, let us denote by $\Omega$ an open bounded set of $\mathbb{R}^{2}$ with smooth boundary $\partial \Omega=\Gamma_{1} \cup \Gamma_{2}$. We assume that over the region $\Omega_{1}$ the plate is sensitive to the change of

\footnotetext{
${ }^{*}$ Supported by a grand of $\mathrm{CNPq}($ Brasil $)$
} 
temperature, while in the complementary part $\Omega_{2}=\Omega \backslash \Omega_{1}$, the plate is indifferent to changes of temperature. Let us denote by $\Gamma_{0}$ the interphace, that is a curve between $\Omega_{1}$ and $\Omega_{2}$, a typical example of $\Omega$ is given by the next picture,

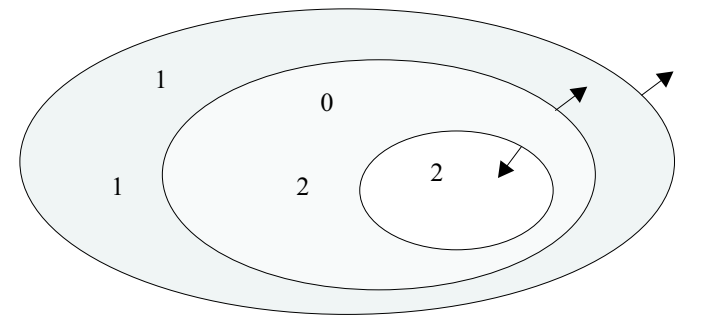

Let us denote by $u$ and $v$ the transverse oscillation over $\Omega_{1}$ and $\Omega_{2}$, respectively and by $\theta$ the difference of temperature, the transmission problem for the thermoelastic plate equation is written as

$$
\begin{aligned}
& \rho_{1} u_{t t}-\gamma_{1} \triangle u_{t t}+\beta_{1} \triangle^{2} u+\mu \triangle \theta=0 \text { in } \quad \Omega_{1} \times \mathbb{R}^{+} \\
& \rho_{0} \theta_{t}-\beta_{0}(k * \triangle \theta)-\mu \Delta u_{t}=0 \text { in } \Omega_{1} \times \mathbb{R}^{+} \\
& \rho_{2} v_{t t}-\gamma_{2} \triangle v_{t t}+\beta_{2} \triangle^{2} v=0 \text { in } \Omega_{2} \times \mathbb{R}^{+}
\end{aligned}
$$

with the following boundary conditions

$$
\begin{gathered}
u=\frac{\partial u}{\partial \nu}=0 \quad \text { on } \quad \Gamma_{1} \times \mathbb{R}^{+}, \quad v=\frac{\partial v}{\partial \nu}=0 \quad \text { on } \quad \Gamma_{2} \times \mathbb{R}^{+} \\
\theta=0 \quad \text { on } \quad \Gamma_{0} \times \mathbb{R}^{+} \text {and } \theta=0 \quad \text { on } \quad \Gamma_{1} \times \mathbb{R}^{+}
\end{gathered}
$$

and the transmission conditions over $\Gamma_{0}$

$$
\begin{gathered}
u=v, \quad \frac{\partial u}{\partial \nu}=\frac{\partial v}{\partial \nu} \\
\beta_{1} \triangle u=\beta_{2} \triangle v \\
\gamma_{1} \frac{\partial u_{t t}}{\partial \nu}-\beta_{1} \frac{\partial \triangle u}{\partial \nu}-\mu \frac{\partial \theta}{\partial \nu}=\gamma_{2} \frac{\partial v_{t t}}{\partial \nu}-\beta_{2} \frac{\partial \triangle v}{\partial \nu}
\end{gathered}
$$

Finally, we prescrive the initial conditions

$$
\begin{gathered}
u(x, 0)=u_{0}(x), \quad u_{t}(x, 0)=u_{1}(x), \quad \theta(x, 0)=\theta_{0}(x) \text { in } \Omega_{1} \\
v(x, 0)=v_{0}(x), \quad v_{t}(x, 0)=v_{1}(x) \text { in } \Omega_{2}
\end{gathered}
$$

The constants $\rho_{0}, \rho_{1}, \rho_{2}, \beta_{0}, \beta_{1}, \beta_{2}, \gamma_{1}, \gamma_{2}, \mu$ are all of them positive. We denote by $k \in C^{1}(0, \infty)$ the relaxation function and by $*$ the convolution product given by

$$
k * \varphi(t)=\int_{0}^{t} k(t-\tau) \varphi(\tau) d \tau .
$$

To be precise in our formulation, let us first introduce the following definition. 
Definition 1.1 Let $k \in L^{1}\left(\mathbb{R}_{+}\right)$, We say that $k$ is a positive definite function when

$$
\int_{0}^{t} \varphi k * \varphi d s \geq 0, \quad \forall t \geq 0, \quad \forall \varphi \in C^{0}\left(\mathbb{R}^{+}\right) .
$$

We say that $k$ is strongly positive definite function when there exists $\delta>0$ such that $k(t)-\delta e^{-t}$ is positive definite.

One important characterization of positive definite function is given in the following theorem, which is proved in [12].

Theorem 1.1 Let $k \in L^{1}\left(\mathbb{R}_{+}\right)$, then $k$ is a positive definite function if and only if

$$
\operatorname{Re} \widehat{k}(i \xi) \geq 0 \quad \text { where } \quad \widehat{k}(\lambda)=\int_{0}^{\infty} k(t) e^{-\lambda t} d t
$$

Remark 1.1 As a consequence of the above theorem we have that $k$ is strongly positive definite if and only if there exists $\delta>0$ such that

$$
\operatorname{Re} \widehat{k}(i \xi) \geq \frac{\delta}{1+\xi^{2}} .
$$

The hypotheses we use to show the existence result and the exponential decay are the following

H1 $k \in C^{2}$ is a strongly positive definite function, satisfying $k(0) \geq 0$

H2 $k$ decays to zero exponentially.

Remark 1.2 Hypotheses $\mathbf{H 2}$ we will use only in the exponential decay. For the existence it is not necessary.

One important point is that the dissipation produced for the Gurtin and Pipkin's law is of indefinite type. That is, the derivative of the energy function can change its signal. In fact, the total energy associated with system (1.1)-(1.10) is given by

$$
\begin{aligned}
E(t)= & \frac{1}{2} \int_{\Omega_{1}} \rho_{1}\left|u_{t}\right|^{2}+\gamma_{1}\left|\nabla u_{t}\right|^{2}+\beta_{1}|\Delta u|^{2}+\rho_{0}|\theta|^{2} d x+ \\
& +\frac{1}{2} \int_{\Omega_{2}} \rho_{2}\left|v_{t}\right|^{2}+\gamma_{2}\left|\nabla v_{t}\right|^{2}+\beta_{2}|\triangle v|^{2} d x .
\end{aligned}
$$

Using the system we can easily verify that

$$
\frac{d}{d t} E(t)=-\beta_{0} \int_{\Omega_{1}}(k * \nabla \theta) \cdot \nabla \theta d x
$$

Note that the right hand side of the above equation, does not have a definite sign. This is because the fact that $k$ be a positive definite function, does not imply that the right hand side of equation (1.12) is positive. In fact, Let us consider the functions

$$
k(t)=e^{-t} \cos t, \quad y(t)=e^{-2 t} .
$$


We have that $k$ satisfies

$$
k \in L^{1}(0, \infty) \quad \text { and } \quad \operatorname{Re} \hat{k}(i \xi) \geq \frac{1}{2\left(1+\xi^{2}\right)}, \quad \forall \xi \in \mathbb{R},
$$

then $k$ is strongly definite positive (see Remark 1.2). On the other hand we have that

$$
\mathcal{Y}(t) \equiv(k * y)(t) \cdot y(t)=\frac{e^{-4 t}}{2}\left[e^{t}(\cos t+\sin t)-1\right],
$$

change of sign. To see this take $t=\frac{\pi}{2}+2 m \pi$ to get $\mathcal{Y}(t)>0$, while for $t=-\frac{\pi}{2}+2 m \pi$ we have $\mathcal{Y}(t)<0$. Therefore $\mathcal{Y}(t)$ change of sign. But $\int_{0}^{T} \mathcal{Y}(t) d t>0$ for any $T>0$.

Integrating (1.12) over $[0, t[$, we get

$$
E(t)=E(0)-\beta_{0} \int_{0}^{t} \int_{\Omega_{1}}(k * \nabla \theta) \cdot \nabla \theta d x
$$

using the fact that $k$ is positive we conclude that $E(t)$ is bounded by $E(0)$. Therefore, system (1.1)-(1.10) is not of dissipative type. Note also that the thermal effect, which produce the indefinite dissipation is active only in one equation. So, we may ask whether the energy associated to system decays or not to zero uniformly as time goes to infinity.

Concerning the literature relative to our problem, we have the work of Fabrizio-LazzariRivera[1], that proves the exponential stability for a thermoelastic plate, when the temperature is active over the whole plate configurated over $\Omega,\left(\Omega_{2}=\emptyset\right)$. Here we follow similar ideias but we have to deal also with estimates over the interphase of the plate which in general produce several problems. In Fatori-Muñoz Rivera [7], the authors consider also the one dimensional thermoelastic system with Gurtin and Pipkin's law for the temperature, also working over the whole domain. In that conditions the authors also proved that the corresponding solution decays exponentially, provided the relaxation function is strongly positive and decays exponentially to zero. Finally, in Fatori-Muñoz Rivera-Lueders [2] the authors consider the transmission problem to the one dimensional thermoelastic system with Gurtin and Pipkin's law active only in a part of the body and proved that the solution decays exponentially, provided the relaxation function is strongly positive definite and decays exponentially to zero. Finally, in [9] the authors consider the transmission problem for the thermoelastic plate equation with the Fourier law. It is proved that the corresponding solution decays exponentially to zero as time goes to infinity, no matter how small is the thermoelastic part (the dissipative part) of the plate.

The main result of this paper is to prove that the partial thermoelastic plate system decays exponentially to zero, no matter the side of the thermoelastic component of the plate $\Omega_{1}$, which produce the thermal dissipation over the plate. The main difference concerning the result in [2] is because of the interphase conditions. For plates such interphase conditions are more complicated than such for one dimensional models, so it is necessary to take more care to estimate terms 
over the interphase of the body configurated over $\Omega$. Concerning the work [9], the system is of hyperbolic-parabolic type because of the Fourier law. This fact, produce a strong dissipation that give an important help to arrive to the exponential decay. In our case, the system, is not dissipative and has not a parabolic part. Therefore to estimate terms in which appears the difference of temperatura is more complicated and demand some techniques of control theory.

The method we use to prove the main result is based on observabilities inequatilies for transmission problems. We also introduce new multipliers which combining with Volterra's method to solve integral equations produce crux estimates to achieve the exponential estability.

The rest of the paper is organized as follows. In section 2 we introduce some notations and establish some results which will be usefull to show the existence of solutions as well as the exponential decay. In section 3 we establish the existence and regularity of solutions. Finally, in section 4 we show the exponential decay.

\section{Notations and Preliminaries}

Let us introduce, the following functional space

$$
\begin{aligned}
& \mathbb{H}^{1}=\left\{(\varphi, \psi) \in H^{1}\left(\Omega_{1}\right) \times H^{1}\left(\Omega_{2}\right) ; \text { satisfying }(2.14)\right\} \\
& \left\{\begin{array}{lll}
\varphi=0 & \text { on } & \Gamma_{1} \\
\psi=0 & \text { on } & \Gamma_{2} \\
\varphi=\psi & \text { on } & \Gamma_{0}
\end{array}\right. \\
& \mathbb{H}^{2}=\left\{(\varphi, \psi) \in\left[H^{2}\left(\Omega_{1}\right) \times H^{2}\left(\Omega_{2}\right)\right] \cap \mathbb{H}^{1} ;\right. \text { satisfying } \\
& \left\{\begin{array}{l}
\frac{\partial \varphi}{\partial \nu}=0 \text { on } \Gamma_{1} \\
\frac{\partial \psi}{\partial \nu}=0 \text { on } \Gamma_{2} \\
\frac{\partial \varphi}{\partial \nu}=\frac{\partial \psi}{\partial \nu} \text { on } \Gamma_{0}
\end{array}\right. \\
& \mathbb{V}^{2}=H^{2}\left(\Omega_{1}\right) \times H^{2}\left(\Omega_{2}\right) \cap \mathbb{H}^{1}, \\
& \mathbb{V}^{4}=H^{4}\left(\Omega_{1}\right) \times H^{4}\left(\Omega_{2}\right) \cap \mathbb{H}^{2} .
\end{aligned}
$$

Lemma 2.1 The space $\mathbb{H}^{1}$ with the inner product

$$
\left\langle\left(\varphi^{1}, \psi^{1}\right),\left(\varphi^{2}, \psi^{2}\right)\right\rangle_{\mathbb{H}^{1}}=\int_{\Omega_{1}}\left(\rho_{1} \varphi^{1} \varphi^{2}+\gamma_{1} \nabla \varphi^{1} \cdot \nabla \varphi^{2}\right) d x+\int_{\Omega_{2}}\left(\rho_{2} \psi^{1} \psi^{2}+\gamma_{2} \nabla \psi^{1} \cdot \nabla \psi^{2}\right) d x
$$

is a Hilbert space. 
Lemma 2.2 The space $\mathbb{H}^{2}$ with the inner product

$$
\left\langle\left(\varphi^{1}, \psi^{1}\right),\left(\varphi^{2}, \psi^{2}\right)\right\rangle_{\mathbb{H}^{2}}=\beta_{1} \int_{\Omega_{1}} \triangle \varphi^{1} \triangle \varphi^{2} d x+\beta_{2} \int_{\Omega_{2}} \triangle \psi^{1} \triangle \psi^{2} d x
$$

is a Hilbert space.

We finish this section establishing the following Lemma whose prove can be found in [14]

Lemma 2.3 Let us suppose that $k \in L^{1}\left(\mathbb{R}^{+}\right)$is a strongly positive definite kernel satisfying $k^{\prime} \in L^{1}\left(\mathbb{R}^{+}\right)$; then we have

$$
\int_{0}^{t}|k * y(\tau)|^{2} d \tau \leq \beta_{0} K \int_{0}^{t} k * y(\tau) y(\tau) d \tau
$$

for any $y \in L_{\text {loc }}^{1}\left(\mathbb{R}^{+}\right)$, where $K=|k|_{1}^{2}+4\left|k^{\prime}\right|_{1}^{2}$ and $\beta_{0}>0$ is such that the function $k(t)-\beta_{0}^{-1} e^{-t}$ is a positive definite kernel.

\section{Existence and uniqueness of solutions}

Here we establish the existence and regularity of weak solutions of (1.1)-(1.10). To this end we assume that the hypotheses $\mathbf{H 1}$ holds. Our starting point is to define what we undertand for weak solution.

Definition 3.1 We say that $(u, v, \theta)$ is a weak solution of the system (1.1)-(1.10) when

$$
\begin{gathered}
(u, v) \in L^{\infty}\left(0, T ; \mathbb{H}^{2}\right) \quad\left(u_{t}, v_{t}\right) \in L^{\infty}\left(0, T ; \mathbb{H}^{1}\right) \\
\theta \in L^{\infty}\left(0, T ; L^{2}\left(\Omega_{1}\right)\right), \quad(k * \theta) \in L^{2}\left(0, T ; H^{1}\left(\Omega_{1}\right)\right)
\end{gathered}
$$

and satisfies the following identities

$$
\begin{aligned}
-\rho_{1} \int_{\Omega_{1}} u_{1}(x) \varphi(x, 0) d x-\gamma_{1} \int_{\Omega_{1}} \nabla u_{1} \cdot \nabla \varphi(x, 0) d x+\rho_{1} \int_{\Omega_{1}} u_{0}(x) \varphi_{t}(x, 0) d x & - \\
-\gamma_{1} \int_{\Omega_{1}} \nabla u_{0} \cdot \nabla \varphi_{t}(x, 0) d x-\rho_{2} \int_{\Omega_{2}} v_{1}(x) \psi(x, 0) d x-\gamma_{2} \int_{\Omega_{2}} \nabla v_{1} \cdot \nabla \psi(x, 0) d x & + \\
+\rho_{2} \int_{\Omega_{2}} v_{0}(x) \psi_{t}(x, 0) d x-\gamma_{2} \int_{\Omega_{2}} \nabla v_{0} \cdot \nabla \psi_{t}(x, 0) d x+\int_{0}^{T} \int_{\Omega_{1}}\left[\rho_{1} u \varphi_{t t}+\gamma_{1} \nabla u \cdot \nabla \varphi_{t t}\right] d x d t & + \\
+\beta_{1} \int_{0}^{T} \int_{\Omega_{1}} \Delta u \Delta \varphi d x d t+\mu \int_{0}^{T} \int_{\Omega_{1}} \theta \Delta \varphi d x d t+\int_{0}^{T} \int_{\Omega_{2}}\left[\rho_{2} v \psi_{t t}+\gamma_{2} \nabla u \cdot \nabla \psi_{t t}\right] d x d t & + \\
+\beta_{2} \int_{0}^{T} \int_{\Omega_{2}} \Delta v \Delta \psi d x d t & =0 \\
\rho_{0} \int_{\Omega_{1}} \theta_{0}(x) \phi(x, 0) d x-\rho_{0} \int_{0}^{T} \int_{\Omega_{1}} \theta \phi_{t} d x d t+\beta_{0} \int_{0}^{T} \int_{\Omega_{1}}(k * \nabla \theta) \cdot \nabla \phi d x d t & + \\
+\mu \int_{0}^{T} \int_{\Omega_{1}} \nabla u_{t} \cdot \nabla \phi d x d t & =0
\end{aligned}
$$


$\forall(\varphi, \psi) \in \mathcal{C}^{2}\left([0, T] ; \mathbb{H}^{2}\right)$ such thah

$$
\begin{aligned}
& \varphi(\cdot, T)=\varphi_{t}(\cdot, T)=\nabla \varphi(\cdot, T)=\nabla \varphi_{t}(\cdot, T)=0 \\
& \psi(\cdot, T)=\psi_{t}(\cdot, T)=\nabla \psi(\cdot, T)=\nabla \psi_{t}(\cdot, T)=0
\end{aligned}
$$

$\forall \phi \in \mathcal{C}^{1}\left([0, T] ; H_{0}^{1}\left(\Omega_{1}\right)\right)$

Using Galerking method, and standard estimates we can show the following theorem.

Theorem 3.1 (Existence of solutions) Let us suppose that hipotheses $\mathbf{H 1}$ holds, if $\left(u^{0}, v^{0}\right) \in$ $\mathbb{H}^{2}, \quad\left(u^{1}, v^{1}\right) \in \mathbb{H}^{1}$ and $\theta^{0} \in H_{0}^{1}\left(\Omega_{1}\right)$. Then, there exist a unique weak solution for (1.1)-(1.10). On the other hand, if $\left(u^{0}, v^{0}\right) \in \mathbb{V}^{4}, \quad\left(u^{1}, v^{1}\right) \in \mathbb{V}^{2}$ and $\theta^{0} \in H^{2}\left(\Omega_{1}\right) \cap H_{0}^{1}\left(\Omega_{1}\right)$ with:

$$
\beta_{1} \triangle u^{0}=\beta \triangle v^{0}, \quad \beta_{1} \frac{\partial \triangle u^{0}}{\partial \nu}=\beta \frac{\partial \triangle v^{0}}{\partial \nu}
$$

then, there exist a unique solution for (1.1)-(1.10), satisfying:

$$
\begin{gathered}
(u, v) \in C\left(\left[0, \infty\left[; \mathbb{V}^{4}\right) \cap C^{1}\left(\left[0, \infty\left[; \mathbb{V}^{2}\right) \cap C^{2}\left(\left[0, \infty\left[; \mathbb{H}^{1}\right)\right.\right.\right.\right.\right.\right. \\
\theta \in C\left(\left[0, \infty\left[; H_{0}^{1}\left(\Omega_{1}\right)\right) \cap C^{1}\left(\left[0, \infty\left[; L^{2}\left(\Omega_{1}\right)\right)\right.\right.\right.\right. \\
(k * \theta) \in L^{2}\left(0, T ; H^{2}\left(\Omega_{1}\right)\right)
\end{gathered}
$$

Remark 3.1 To show the existence of weak solution we only need that the strongly positive function $k$ be in $C^{0}$, and $k(0)>0$. Instead, to get the regularity result we need hypotheses $\mathbf{H 1}$.

\section{Exponential decay}

In this section we will assume that the domain $\Omega$ has the following geometric property: There exists $x_{0} \in \mathbb{R}^{2}$ such that the function $m(x)=x-x_{0}$ satisfies:

$$
\begin{array}{ll}
m \cdot \nu \geq \delta_{0} \quad>0 & \text { in } \quad \Gamma_{0} \\
m \cdot \nu \leq 0 & \text { in } \Gamma_{2}
\end{array}
$$

for $\delta_{0}>0$.

To show that the solution decays exponentially to zero as time goes to infinity. we introduce the following functions

$$
U(x, t)=u(x, t) e^{\eta t}, \quad \Theta(x, t)=\theta(x, t) e^{\eta t}, \quad \text { and } \quad V(x, t)=v(x, t) e^{\eta t},
$$

Therefore, to prove the exponential decay, we only have to prove that the above functions are uniformly bounded with respect to the time for $\eta$ small enought. To do this, let us differentiating 
with respect to the time to get

$$
\begin{aligned}
U_{t}=\eta U+e^{\eta t} u_{t}, & U_{t t}=2 \eta U_{t}+e^{\eta t} u_{t t}-\eta^{2} U \\
V_{t}=\eta V+e^{\eta t} v_{t}, & V_{t t}=2 \eta V_{t}+e^{\eta t} v_{t t}-\eta^{2} V \\
\Theta_{t}=\eta \Theta+e^{\eta t} \theta_{t}, & \tilde{k} * \triangle \Theta=e^{\eta t}(k * \triangle \theta) .
\end{aligned}
$$

From the above identities and using equations (1.1)-(1.10), we see that the functions $(U, \Theta, V)$ satisfies the following system

$$
\begin{aligned}
& \rho_{1} U_{t t}-\gamma_{1} \triangle U_{t t}+\beta_{1} \triangle^{2} U+\mu \triangle \Theta=\mathcal{P}, \quad \text { in } \quad \Omega_{1} \times \mathbb{R}^{+} \\
& \rho_{0} \Theta_{t}-\beta_{0}(\tilde{k} * \triangle \Theta)-\mu \triangle U_{t}=\mathcal{Q}, \quad \text { in } \Omega_{1} \times \mathbb{R}^{+} \\
& \rho_{2} V_{t t}-\gamma_{2} \triangle V_{t t}+\beta_{2} \triangle^{2} V=\mathcal{R}, \quad \text { in } \Omega_{2} \times \mathbb{R}^{+}
\end{aligned}
$$

with the following boundary conditions:

$$
\begin{gathered}
U=\frac{\partial U}{\partial \nu}=0 \quad \text { on } \quad \Gamma_{1} \times \mathbb{R}^{+}, \quad V=\frac{\partial V}{\partial \nu}=0 \quad \text { on } \quad \Gamma_{2} \times \mathbb{R}^{+} \\
\Theta=0 \quad \text { on } \quad \Gamma_{0} \times \mathbb{R}^{+} \quad \text { and } \quad \Theta=0 \quad \text { on } \quad \Gamma_{1} \times \mathbb{R}^{+}
\end{gathered}
$$

Finally, the couple $(U, V)$ must verify the following transmission conditions:

$$
\begin{gathered}
U=V, \quad \frac{\partial U}{\partial \nu}=\frac{\partial V}{\partial \nu} \\
\beta_{1} \triangle U=\beta_{2} \triangle V \\
\gamma_{1}\left\{\frac{\partial U_{t t}}{\partial \nu}-2 \eta \frac{\partial U_{t}}{\partial \nu}+\eta^{2} \frac{\partial U}{\partial \nu}\right\}-\beta_{1} \frac{\partial \triangle U}{\partial \nu}-\mu \frac{\partial \Theta}{\partial \nu}=\gamma_{2}\left\{\frac{\partial V_{t t}}{\partial \nu}-2 \eta \frac{\partial V_{t}}{\partial \nu}+\eta^{2} \frac{\partial V}{\partial \nu}\right\}-\beta_{2} \frac{\partial \triangle V}{\partial \nu}
\end{gathered}
$$

in $\Gamma_{0} \times \mathbb{R}^{+}$; and initial conditions:

$$
\begin{gathered}
U(x, 0)=u_{0}(x), \quad U_{t}(x, 0)=u_{1}(x)+\eta u_{0}(x) \quad \text { in } \Omega_{1} \\
\Theta(x, 0)=\theta_{0}(x) \quad \text { in } \quad \Omega_{1} \\
V(x, 0)=v_{0}(x), \quad V_{t}(x, 0)=v_{1}(x)+\eta v_{0}(x) \quad \text { in } \quad \Omega_{2}
\end{gathered}
$$

where $\mathcal{P}, \mathcal{Q}$ and $\mathcal{R}$ are given by:

$$
\begin{aligned}
\mathcal{P} & =2 \eta \rho_{1} U_{t}-\rho_{1} \eta^{2} U+\gamma_{1} \eta^{2} \triangle U-2 \gamma_{1} \eta \triangle U_{t}, \\
\mathcal{Q} & =\rho_{0} \eta \Theta-\mu \eta \triangle U, \\
\mathcal{R} & =2 \eta \rho_{2} V_{t}-\rho_{2} \eta^{2} V+\gamma_{2} \eta^{2} \triangle V-2 \gamma_{2} \eta \triangle V_{t} .
\end{aligned}
$$


Let us introduce the energy function $\mathcal{E}(t)$ associated with the above equations,

$$
\begin{aligned}
\mathcal{E}(t)= & \frac{1}{2} \int_{\Omega_{1}} \rho_{1}\left|U_{t}\right|^{2}+\gamma_{1}\left|\nabla U_{t}\right|^{2}+\beta_{1}|\triangle U|^{2}+\rho_{0}|\Theta|^{2} d x+\frac{\eta^{2}}{2} \int_{\Omega_{1}} \rho_{1}|U|^{2}+\gamma_{1}|\nabla U|^{2} d x \\
& +\frac{\rho_{2}}{2} \int_{\Omega_{2}}\left|V_{t}\right|^{2}+\gamma_{2}\left|\nabla V_{t}\right|^{2}+\beta_{2}|\triangle V|^{2} d x+\frac{\eta^{2}}{2} \int_{\Omega_{2}} \rho_{2}|V|^{2}+\gamma_{2}|\nabla V|^{2} d x .
\end{aligned}
$$

To show the exponential decay of $E(t)$ it is enough to show that $\mathcal{E}(t)$ is uniformly bounded for any $t>0$. To this end, we start with the following Lemma.

Lemma 4.1 Let us suppose that the initial data satisfy $\left(u_{0}, v_{0}\right) \in \mathbb{V}^{4},\left(u_{1}, v_{1}\right) \in \mathbb{V}^{2}$ e $\theta_{0} \in \mathbb{W}^{2}$. Then, there exist positive constant $c$ and $\beta_{0}$ satisfying

$$
\frac{d}{d t} \mathcal{E}(t) \leq-\beta_{0} \int_{\Omega_{1}}(\tilde{k} * \nabla \Theta) \cdot \nabla \Theta d x+c \eta \mathcal{E}(t) .
$$

Proof: Multiplying equation (4.16) by $U_{t},(4.17)$ by $\Theta$ and (4.18) by $V_{t}$ and summing up the product result our conclusion follows.

Theorem 4.1 Let us take $\left(u_{0}, v_{0}\right) \in \mathbb{V}^{4},\left(u_{1}, v_{1}\right) \in \mathbb{V}^{2}$ and $\theta_{0} \in \mathbb{H}^{2}\left(\Omega_{1}\right) \cap \mathbb{H}^{1}\left(\Omega_{1}\right)$. If

$$
\rho_{1} \geq \rho_{2}, \quad \gamma_{1} \geq \gamma_{2} \quad \text { e } \beta_{1} \leq \beta_{2},
$$

then the solution of system (1.1)-(1.10) decays exponentially to zero as time goes to infinity. That is, there exist positive constans $C>0, \lambda>0$, such that

$$
E(t) \leq C E(0) e^{-\lambda t}
$$

Proof: Let us denote by $\mathcal{K} U=m \cdot \nabla U-\frac{1}{2} U$, multiplying equation (4.16) by $\mathcal{K} U$, equation (4.18) by $\mathcal{K} V$, applying hypotheses (4.27) and using integration by parts we get

$$
\begin{aligned}
\frac{d}{d t} I(t) \leq & \frac{\beta_{1}}{2} \int_{\Gamma_{1}}(m \cdot \nu)|\triangle U|^{2} d x+\frac{\rho_{1} \eta^{2}}{2} \int_{\Gamma_{0}}(m \cdot \nu)|U|^{2} d x+\frac{\gamma_{1} \eta^{2}}{2} \int_{\Gamma_{0}}(m \cdot \nu)|\nabla U|^{2} d x \\
& -\frac{3 \rho_{1}}{2} \int_{\Omega_{1}}\left|U_{t}\right|^{2} d x-\frac{\gamma_{1}}{2} \int_{\Omega_{1}}\left|\nabla U_{t}\right|^{2} d x-\frac{\beta_{1}}{2} \int_{\Omega_{1}}|\triangle U|^{2} d x-\frac{3 \rho_{2}}{2} \int_{\Omega_{2}}\left|V_{t}\right|^{2} d x \\
& -\frac{\gamma_{2}}{2} \int_{\Omega_{2}}\left|\nabla V_{t}\right|^{2} d x-\frac{\beta_{2}}{2} \int_{\Omega_{2}}|\triangle V|^{2} d x+\mu \int_{\Omega_{1}} \nabla \Theta \cdot \nabla(m \cdot \nabla U) d x \\
& +\frac{\mu}{2} \int_{\Omega_{1}} \Theta \triangle U d x+\eta c_{0} \mathcal{E}(t),
\end{aligned}
$$

where

$$
\begin{gathered}
I(t)=\rho_{1} \int_{\Omega_{1}} U_{t} \mathcal{K} U+\gamma_{1} \int_{\Omega_{1}} \nabla U_{t} \cdot \nabla \mathcal{K} U d x-\frac{\eta \rho_{1}}{2} \int_{\Omega_{1}}|U|^{2} d x-\frac{\eta \gamma_{1}}{2} \int_{\Omega_{1}}|\nabla U|^{2} d x+\rho_{2} \int_{\Omega_{2}} V_{t} \mathcal{K} V \\
+\gamma_{2} \int_{\Omega_{2}} \nabla V_{t} \cdot \nabla \mathcal{K} V d x-\frac{\eta \rho_{2}}{2} \int_{\Omega_{2}}|V|^{2} d x-\frac{\eta \gamma_{2}}{2} \int_{\Omega_{2}}|\nabla V|^{2} d x .
\end{gathered}
$$


The main problem in the above inequality is with the term $\mu \int_{\Omega_{1}} \nabla \Theta \cdot \nabla(m \cdot \nabla U) d x$, which is not possible to estimate in terms of the first order energy $\mathcal{E}(t)$. For this reason we have to construct a funcional, whose derivative has such term in order to eliminate it. The functional we consider to do this task is the following,

$$
\mathcal{M}(t)=\rho_{0} \int_{\Omega_{1}} \Theta m_{i} \frac{\partial}{\partial x_{i}}(\tilde{k} * \Theta) d x-\mu \int_{\Omega_{1}} \triangle U m_{i} \frac{\partial}{\partial x_{i}}(\tilde{k} * \Theta) d x
$$

That is to say, let us multiply equation (4.17) by $m_{i} \frac{\partial}{\partial x_{i}}(\tilde{k} * \Theta)$. Therefore for $\eta>0$ small enough, we have that there exist positive constants $c_{1}, c_{2}, c_{3}$, such that:

$$
\begin{aligned}
\frac{d}{d t} \mathcal{M}(t) \leq & -\frac{\beta_{0} \delta_{0}}{2} \int_{\Gamma_{0}}\left|\tilde{k} * \frac{\partial \Theta}{\partial \nu}\right|^{2} d x+\frac{\beta_{0}}{2} \int_{\Gamma_{1}}(m \cdot \nu)\left|\tilde{k} * \frac{\partial \Theta}{\partial \nu}\right|^{2} d x-c_{1} \int_{\Omega_{1}}|\Theta|^{2} d x \\
& -\mu \tilde{k}(0) \int_{\Omega_{1}} \nabla \Theta \cdot \nabla(m \cdot \nabla U) d x+c_{2} \int_{\Omega_{1}}|\tilde{k} * \nabla \Theta|^{2}+\eta c_{3} \mathcal{E}(t) .
\end{aligned}
$$

Where we have used that

$$
\int_{\Omega_{1}} \nabla \Theta \cdot \nabla(m \nabla U) d x=\int_{\Omega_{1}}(m \cdot \nabla \Theta) \triangle U d x .
$$

To simplifly notations, let us introduce the functional $G(t)=I(t)+\frac{1}{\tilde{k}(0)} \mathcal{M}(t)$. For $\eta>0$ small enough there exist positive constants $c_{0}, c_{1}, c_{2}$ such that

$$
\begin{aligned}
\frac{d}{d t} G(t) \leq & \frac{\beta_{1} c_{0}}{2} \underbrace{\int_{\Gamma_{1}}|\triangle U|^{2} d x}_{:=J_{1}}+\frac{\beta_{0} c_{0}}{2 \tilde{k}(0)} \underbrace{\int_{\Gamma_{1}}\left|\tilde{k} * \frac{\partial \Theta}{\partial \nu}\right|^{2} d x}_{:=J_{2}}-\frac{\beta_{0} \delta_{0}}{2 \tilde{k}(0)} \int_{\Gamma_{0}}\left|\tilde{k} * \frac{\partial \Theta}{\partial \nu}\right|^{2} d x \\
& -c_{1} \mathcal{E}(t)+c_{2} \int_{\Omega_{1}}|\tilde{k} * \nabla \Theta|^{2} d x+\frac{\mu^{2}}{4 \beta_{1}} \int_{\Omega_{1}}|\Theta|^{2} d x .
\end{aligned}
$$

From the above inequality, we see that our next problem, is to estimate the boundary terms $J_{1}$ and $J_{2}$. To do this, we apply an observability technique which consist in to use some multipliers at the boundary, such as $h=\left(h_{1}, h_{2}\right) \in\left[C^{2}(\Omega)\right]^{n}$ defined by

$$
h(x)=\left\{\begin{array}{rlc}
-\nu(x) & \text { se } & x \in \Gamma_{1} \\
0 & \text { se } & x \in B_{\delta}\left(\Omega_{2}\right) .
\end{array}\right.
$$

where

$$
B_{\delta}\left(\Omega_{2}\right)=\left\{x \in \Omega, \operatorname{dist}\left(x, \Omega_{2}\right) \leq \delta\right\} .
$$

Let us introduce the functional

$$
\begin{aligned}
H(t)= & \rho_{1} \int_{\Omega_{1}} U_{t}(h \cdot \nabla U) d x+\gamma_{1} \int_{\Omega_{1}} \nabla U_{t} \cdot \nabla(h \cdot \nabla U) d x \\
& +\frac{1}{\tilde{k}(0)}\left\{\rho_{0} \int_{\Omega_{1}} \Theta h_{i} \frac{\partial}{\partial x_{i}}(\tilde{k} * \Theta) d x-\mu \int_{\Omega_{1}} \triangle U h_{i} \frac{\partial}{\partial x_{i}}(\tilde{k} * \Theta) d x\right\},
\end{aligned}
$$


Multiplying equation (4.16) by $(h \cdot \nabla U)$ and equation (4.17) by $h_{i} \frac{\partial}{\partial x_{i}}(\tilde{k} * \Theta)$. Summing up the product results, we get for $\eta>0$ small enough that there exist positive constants $C_{i}$ with $i=1,2, \ldots, 5$ such that:

$$
\begin{aligned}
\frac{d}{d t} H(t) \leq & -\frac{\beta_{1}}{2} \int_{\Gamma_{1}}|\triangle U|^{2} d x-\frac{\beta_{0}}{2 \tilde{k}(0)} \int_{\Gamma_{1}}\left|\tilde{k} * \frac{\partial \Theta}{\partial \nu}\right|^{2} d x-C_{1} \int_{\Omega_{1}}\left|U_{t}\right|^{2} d x+\eta C_{2} \int_{\Omega_{1}}\left|\nabla U_{t}\right|^{2} d x \\
& -C_{3} \int_{\Omega_{1}}|\triangle U|^{2} d x-C_{4} \int_{\Omega_{1}}|\Theta|^{2} d x+C_{5} \int_{\Omega_{1}}|\tilde{k} * \nabla \Theta|^{2} d x .
\end{aligned}
$$

To simplify once more, let us denote by $F(t)$ the function $F(t)=G(t)+\epsilon_{0} H(t)$. So we have that for $\epsilon_{0}>0$ large enough and for $\eta>0$ small, there exists a positive constant, we denote by $c_{i}$ with $i=1,2, \ldots 5$, such that

$$
\begin{aligned}
\frac{d}{d t} F(t) \leq & -c_{1} \int_{\Gamma_{1}}|\triangle U|^{2} d x-c_{2} \int_{\Gamma_{1}}\left|\tilde{k} * \frac{\partial \Theta}{\partial \nu}\right|^{2} d x-c_{3} \int_{\Gamma_{0}}\left|\tilde{k} * \frac{\partial \Theta}{\partial \nu}\right|^{2} d x \\
& -c_{4} E(t)+c_{5} \int_{\Omega_{1}}|\tilde{k} * \nabla \Theta|^{2} d x .
\end{aligned}
$$

Let us define the Lyapunov's functional $\mathcal{L}$, given by

$$
\mathcal{L}(t)=N \mathcal{E}(t)+F(t)
$$

where $N$ denotes a large positive constant to be fixed later. Combining Lemma 4.1 and inequality (4.28) and using Lemma 2.3 we conclude that:

$$
\begin{aligned}
\frac{d}{d t} \mathcal{L}(t) & \leq-\beta_{0} N \int_{\Omega_{1}}(\tilde{k} * \nabla \Theta) \cdot \nabla \Theta d x+c_{5} \int_{\Omega_{1}}|\tilde{k} * \nabla \Theta|^{2} d x-\left(c_{4}-\eta c N\right) E(t, U, \Theta, V)- \\
& -c_{1} \int_{\Gamma_{1}}|\triangle U|^{2} d x-c_{2} \int_{\Gamma_{1}}\left|\tilde{k} * \frac{\partial \Theta}{\partial \nu}\right|^{2} d x-c_{3} \int_{\Gamma_{0}}\left|\tilde{k} * \frac{\partial \Theta}{\partial \nu}\right|^{2} d x
\end{aligned}
$$

Integrating over $[0, T]$ we get

$$
\begin{aligned}
\mathcal{L}(t) \leq & \mathcal{L}(0)-\beta_{0} N \int_{0}^{t} \int_{\Omega_{1}}(\tilde{k} * \nabla \Theta) \cdot \nabla \Theta d x d \tau+c_{5} \int_{0}^{t} \int_{\Omega_{1}}|\tilde{k} * \nabla \Theta|^{2} d x d \tau- \\
& -\left(c_{4}-\eta c N\right) \int_{0}^{t} \mathcal{E}(\tau) d \tau-c_{1} \int_{0}^{t} \int_{\Gamma_{1}}|\triangle U|^{2} d x d \tau- \\
& -c_{2} \int_{0}^{t} \int_{\Gamma_{1}}\left|\tilde{k} * \frac{\partial \Theta}{\partial \nu}\right|^{2} d x d \tau-c_{3} \int_{0}^{t} \int_{\Gamma_{0}}\left|\tilde{k} * \frac{\partial \Theta}{\partial \nu}\right|^{2} d x d \tau .
\end{aligned}
$$

Using Young's inequality we can to prove that for $N$ large enough, there exist positive constant $C_{1}, C_{2}$ such that

$$
e^{2 \eta t} E(t) \leq 2 \mathcal{E}(t) \leq C_{1} \mathcal{L}(t)+C_{2} \int_{\Omega_{1}}|\tilde{k} * \nabla \Theta|^{2} d x
$$


Combining (4.29) and (4.30) we conclude that for $N$ large enough and $\eta$ small enough we have:

$$
E(t) \leq C_{1} \mathcal{L}(0) e^{-2 \eta t} \leq C E(0) e^{-2 \eta t} .
$$

From where our conclusions follows.

Remark 4.1 : As a final remark, we obtain from (4.29) the regularity result

$$
\frac{\partial(k * \theta)}{\partial \nu} \in L^{2}\left(0, T ; L^{2}\left(\Gamma_{0} \cup \Gamma_{1}\right)\right), \quad \triangle u \in L^{2}\left(0, T ; L^{2}\left(\Gamma_{1}\right)\right)
$$

which can not be obtained as a consequence of the regularity result of the solution.

\section{References}

[1] M. Fabrizio, B. Lazzari and J.E. Muñoz Rivera. Asymptotic behaviour of thermoelastic plate of weakly hyperbolic type, Differential and Integral Equations, 13(2000), 1347-1370.

[2] L.H. Fatori J.E. Muñoz Rivera and E. Lueders. Transmission problem for hyperbolic thermoelastic system. Journal of Thermal Stresses Volume 26-7, 739-764 (2003).

[3] M.E. Gurtin and A.C. Pipkin. A general theory of heat conduction with finite wave speeds. Arch. Rational Mech. Anal 31,113-126 (1968).

[4] J. U. Kim, On the energy decay of a linear thermoelastic plates. SIAM J. Math. Anal., Vol. 23, (1992) 889-899.

[5] Z.Y. Liu and M. Renardy, A note on the equations of a thermoelastic plate. Appl. Math. Lett. 8(3)(1995),1-6.

[6] W. Liu and G. Williams, The Exponential Stability of the Problem of Transmission of the Wave Equations. Bull. Austral. Math. Soc., vol.57(1998),305-327.

[7] J. E. Muñoz Rivera and L. Harue Fatori, Transmission problem for hyperbolic thermoelastic system. Quarterly of Applied Mathematics. Volume LIX, Número 3, páginas 441 - 458 (2001).

[8] J. E. Muñoz Rivera and H. Portillo Oquendo, The Transmission problem of thermoelastic beams. Journal of Thermal Stresses, 24 (2001),1137-1158.

[9] J. E. Muñoz Rivera and H. Portillo Oquendo, A transmission problem for thermoelastic plates. Quarterly of Applied Mathematics. Volume 62, Número 2, páginas 273 - 293 (2004). 
[10] J. E. Muñoz Rivera and Y. Shibata, A linear thermoelastic plate equations with Dirichlet boundary condition. Math. Met. Appl. Sc. ,20(11) (1997), 915-932.

[11] J. E. Muñoz Rivera and R. Racke, Smoothing properties, decay and global existence of solutions to nonlinear coupled systems of thermoelastic type. Siam J. Math. Anal. 26(6) (1995), 1547-1563.

[12] J.A. Nohel and D.F. Shea, Frequency domain methods for Volterra equations. Adv. Math. Vol. 22, pp 278-304, 1976.

[13] Shibata, Y. On the exponential decay of thermoelastic plate. Comp. Appl. Math., Vol. 13, pp 81-102, 1994.

[14] O. J. Staffans; On a nonlinear hyperbolic Volterra Equation. Siam J. Math, Anal. 11, 5, 793-812 (1980). 